\title{
Bacillus Calmette-Guerin vaccine and bladder cancer incidence: Scoping literature review and preliminary analysis
}

\author{
Sabrina Trigo ${ }^{1}$, Kaitlin Gonzalez ${ }^{1}$, Livio Di Matteo ${ }^{2}$, Asmaa Ismail ${ }^{1}$, Hazem Elmansy ${ }^{1}$, \\ Walid Shahrour ${ }^{1}$, Owen Prowse ${ }^{1}$, Ahmed Kotb ${ }^{1}$ \\ ${ }^{1}$ Northern Ontario School of Medicine, Thunder Bay, ON, Canada; \\ 2 Department of Economics, Lakehead University, Thunder Bay, ON, Canada.
}

\begin{abstract}
Summary Background: The Bacillus Calmette-Guerin (BCG) vaccine has long been used for the prevention of tuberculosis (TB) around the world. BCG is also used as an immunotherapy agent for the treatment of non-muscle invasive urinary bladder cancer. This scoping literature review and preliminary data analysis aims to summarize the literature correlating infantile BCG vaccination with the incidence of future bladder cancer.

Methods: Studies were identified by a formal literature search of MEDLINE and Cochrane Central Registrar of Controlled Trials following PRISMA guidelines. Preliminary data analysis was conducted on publicly accessible data summarizing the impact of gender, BCG vaccination, and socio-economic effects on crude and age-standardized rates of bladder cancer.

Results: As part of our analysis, preliminary regression models demonstrated BCG vaccination status, gender, and socio-economic status to have statistically significant effects on crude and age-standardized rates of bladder cancer incidence. BCG vaccination was associated with a 35-37\% lower age-standardized rate of bladder cancer incidence.

Conclusions: There is very little literature examining the relationship between prior BCG vaccination and rates of bladder cancer incidence. Our limited data analysis indicates that a relationship does exist between infantile BCG vaccination and later bladder cancer development, although extensive future investigation is needed in this area.
\end{abstract}

KEY WORDS: BCG; Bladder cancer; BCG vaccine; Cancer prevention.

Submitted 28 December 2020; Accepted 29 December 2020

\section{INTRODUCTION}

The Bacillus Calmette-Guerin (BCG) vaccine is a live attenuated strain of Mycobacterium bovis that has been used for the prevention of tuberculosis (TB) (1). As part of the World Health Organization Global Expanded Immunization Program, since the mid 1900's, the BCG vaccine has remained as one of the most widely used vaccines around the world $(2,3)$. Implementation of this vaccine has resulted in significantly decreased rates of TB globally $(2,3)$. In most western countries, immunization campaigns in the early $20^{\text {th }}$ century have led to near eradication of $\mathrm{TB}$ and thus discontinuation of BCG vaccination programs (4-6). Currently, select countries, primarily in
South America and East Asia continue to vaccinate against $\mathrm{TB}$, while other countries rely on targeted vaccination of only high-risk groups (5-7). For more than thirty years; BCG material has also served as the standard intravesical immunotherapy agent in the treatment of non-muscle invasive urinary bladder cancer (NMIBC) following transurethral resection (TUR) $(8,9)$. This combined approach to NMIBC offers the most successful treatment for bladder cancer to date $(8,10)$. In the past, the BCG vaccine was examined for its anti-cancer properties, which even until today are not well understood. However, it is speculated that BCG has anti-neoplastic properties through its ability to upregulate immunologic cytokine expression within the urinary bladder and induce a form of trained immunologic memory (11). Presently, urinary bladder neoplasms remain in the top 10 most common cancers around the world (12). Furthermore, incidence rates of bladder cancer have been increasing over the past twenty years, and the rates of bladder cancer are higher in first world countries (13).

In these same countries, TB vaccination programs have ceased or target high-risk individuals, including immigrants from TB endemic areas and a select few indigenous populations living in highly crowded conditions (4-6). The role of BCG in the preventing recurrence of NMIBC when combined with TUR is well established and standard of care. However, it is presently unknown if the BCG vaccine given in early childhood actually serves as a protective mechanism for the development of bladder cancer in later life and whether or not increased rates of bladder cancer correlate with decreased BCG vaccination in various nations.

This scoping literature review serves to summarize what is presently known of the connection between bladder cancer and BCG vaccination, and whether previous BCG immunization has any protective mechanisms for bladder cancer development in later life. Results from this review will inform future research endeavors in the area of urinary bladder cancer prevention.

\section{Methods}

\section{Rationale}

The rationale of this scoping literature review is to establish whether there is existing literature examining the link

No conflict of interest declared. 
between the BCG vaccine and rates of bladder cancer. We also aimed to summarize the impact of gender, BCG vaccination, and socio-economic effects on the crude and age-standardized rates of bladder cancer. The relationship between crude and age standardized rates of bladder cancer incidence versus the aforementioned variables and other confounders was examined to investigate if an inverse relationship between current bladder cancer incidence and current use of BCG vaccines is related.

\section{Retrieval of studies}

A comprehensive virtual literature search was conducted in MEDLINE and the Cochrane Central Registrar of Controlled Trials using PRISMA guidelines for scoping literature reviews. A protocol was created for this literature search but was not submitted for publication. This article is a review and consists solely of a literature search and analysis of publicly accessible information. It did not include patient data, thus was exempt from Research ethics board approval. The literature search was performed on September 6, 2020. The search was conducted for articles pertaining to the BCG vaccine and bladder cancer published in the English language. Search terms used included "bladder cancer" or "urinary bladder neoplasm" and "bcg vaccine" or "bcg" or "mycobacterium bovis". Two Reviewers independently reviewed titles, abstracts, full articles, and reference lists of articles selected for full-text review. Decision to include an article at any stage of the review was made by consensus, if there was a discrepancy a third reviewer served as a tiebreaker. At each stage of review, all studies were carefully assessed for relevance.

\section{Inclusion and exclusion criteria}

In this analysis, we used the following inclusion criteria: (1) original research from randomized controlled trials, cohort studies, case-control studies, observational studies, or correlational studies; (2) patients vaccinated with BCG prior to bladder cancer diagnosis; (3) reported bladder cancer incidence or associated mortality. We did not consider review articles and guidelines or studies that included unvaccinated patients where the outcome of interest was not a bladder neoplasm.

\section{External data analysis}

Both linear and log-linear regression analysis was conducted using data obtained from the Cancer Incidence in Five Continents, Vol. 10, the BCG World Atlas, and the International Monetary Fund (IMF) World Economic Outlook Database to describe the impact of BCG vaccination on bladder cancer rates. Next, we obtained the age standardized and crude bladder cancer incidence rates for females and males from 70 countries between 2003 and 2007. Additional variables of interest were subsequently assigned to these countries, which included the following: If the country has current Bacillus-CalmetteGuerin (BCG) vaccination programs for TB (1 yes, 0 no); whether the national share of 1-year-olds vaccinated for BCG exceeded $80 \%$ ( 1 yes, 0 no); if the country never had a vaccination program or had or discontinued universal BCG vaccination (1 yes, 0 no); the national share of 1-year-olds immunized for BCG in both 1985 and 2015 (\%); the median per capita Gross Domestic Product
(GDP) from 2003-07; the median unemployment rate from 2003-07; and, the median government spending to GDP (\%) from 2003-07. If we accept that current rates of international BCG vaccination are an acceptable proxy for past levels, then the using country having current BCG vaccination for TB (1 yes, 0 no) and National Share of 1 Y Old's Immunized (BCG) (WHO) in $2015>80 \%$ can be used as the TB vaccine variables. As well, we can also use another variable defined as Country Never Had or Discontinued Universal BCG (1 yes, 0 no). This variable may be better at picking up the lagged effects over time of $\mathrm{TB}$ vaccine use.

\section{Results}

\section{Literature search}

Our search resulted in 680 articles. Forty-two duplicates were identified and removed. Six hundred and thirtyeight articles underwent title screening. Subsequently, sixteen abstracts were reviewed, followed by 3 full-text articles and corresponding reference lists. Three articles were found to be of relevance to our work, however only one publication examined the relationship between BCG vaccination and bladder cancer. The identified study examined the relationship between childhood BCG vaccination and the subsequent development of lung cancer as the primary outcome of interest and bladder cancer as a secondary outcome. This article found BCG vaccination to not be associated with a decreased rate of bladder cancer (HR, 1.34; 95\% CI, 0.22-8.03). The study demonstrated that the rate of lung cancer was significantly lower in those who received the BCG vaccine compared to those who had received the placebo (18.2 vs 45.4 cases per 100000 person-years; hazard ratio, 0.38; 95\% CI, 0.20-0.74; $\mathrm{p}=.005$ ), controlling for sex, region, alcohol overuse, smoking, and tuberculosis. No other types of malignant neoplasms were significantly different between the 2 groups; notably, leukemia and lymphoma rates were similar in the BCG group vs placebo group (HR, 0.80; 95\% CI, 0.35-1.82). Ten individuals had a second malignant neoplasm, including cancers of the skin, breast, uterus, ovary, and pancreas and leukemia. The study was a retrospective review of a clinical trial that had assigned participants to a vaccine group using systematic stratification of participants based on school age, district, and sex. The participants $(n=2963)$ were subsequently randomized by alternation. The original study occurred at 9 sites in 5 US states in between 1935 and 1998 and involved indigenous and Alaskan school children with no prior evidence of tuberculosis infection. One cohort received a single intradermal BCG injection and the other, a saline placebo. The outcome of interest was diagnosis of cancer following BCG vaccination.

\section{Data exploration}

Age standardized and crude bladder cancer incidence rates for males and females for 70 international geographic units for the period 2003-07 were obtained and additional variables assigned for these national units. These variables include: country has current bcg vaccination for tuberculosis ( 1 yes, 0 no), country never had or discontinued universal BCG ( 1 yes, 0 no), national share of $1 \mathrm{y}$ 
olds immunized (BCG) (WHO) in $2015>80 \%$, BCG immunization coverage among 1-year-olds (WHO 2017) (\%) in 1985, BCG immunization coverage among 1-yearolds (WHO 2017) (\%) in 2015, Median Per Capita GDP 2003-07, Median Unemployment Rate 2003-07 and Median Government Spending to GDP (\%) 2003-07.

Given that bladder cancer is primarily a disease of age and TB vaccination occurs early in life, one can hypothesis that any relationship between rates of vaccination and bladder cancer incidence is a lagged one with current bladder cancer incidence rates a function of rates of vaccination and coverage 30 to 50 years ago. On the other hand, current rates of vaccination may be a suitable proxy for past rates and useful as a determining variable. As well, for some of these variables, it was not possible to assign a value. Therefore, while the upper bound size of the dataset is 140 observations, some of the analysis would inevitably be restricted to dataset size of just over half the total number of observations.

In any event, the analysis is exploratory and very preliminary. If we accept that current rates of international BCG vaccination are an acceptable proxy for past levels, then using country has current BCG vaccination for tuberculosis ( 1 yes, 0 no) and national share of 1 y olds immunized (BCG) (WHO) in $2015>80 \%$ can be used as the TB vaccine variables. We can look at the relationship between crude and age standardized rates of bladder cancer incidence versus these variables as well as other confounders to see if an inverse relationship between current bladder cancer incidence and current use of $\mathrm{TB}$ vaccine. As well, we can also use another variable defined as Country Never Had or Discontinued Universal BCG (1 yes, 0 no). This variable may be better at picking up the lagged effects over time of TB vaccine use. As well, a variable was generated called National Share of 1 y olds immunized (BCG) (WHO 2017) in $1985>50 \%$ ( 1 if $>50 \%, 0$ if $<50 \%$ or country never had or discontinued universal BCG $=1$ ). This exploratory analysis will be confined to those variables for which the most observations are available.

In terms of results, locally weighted scatterplot smoothing (LOWESS) univariate plots of age standardized bladder cancer incidence versus whether there is current universal BCG vaccination [county has current BCG vaccination for tuberculosis ( 1 yes, 0 no)] is quite negative for males and slightly negative for females (Figure 1). Plots of the age-standardized incidence rates versus GDP appears to show a hump-shaped relationship for both males and females suggesting rising incidence as GDP rises and then a decline at higher levels of GDP (Figure 2 ). This can be interpreted as rising incidence during rising socio-economic status as captured by per capita GDP but then a leveling off and decline. However, these plots do not control for confounding factors.

Regression analysis is conducted regressing both crude and age-standardized bladder cancer rates controlling for whether male or female incidence, GDP and GDP squared (to model the potential hump-shaped effect of the economic status variable) and BCG vaccination variables. The regressions are for linear and log-linear models. In the linear models for the determinants of both cruse and age standardized incidence, the first set uses current universal
Figure 1.

Age standardized bladder cancer incidence rate (2003-07) versus percent of one year old getting BCG vaccination in 1985.
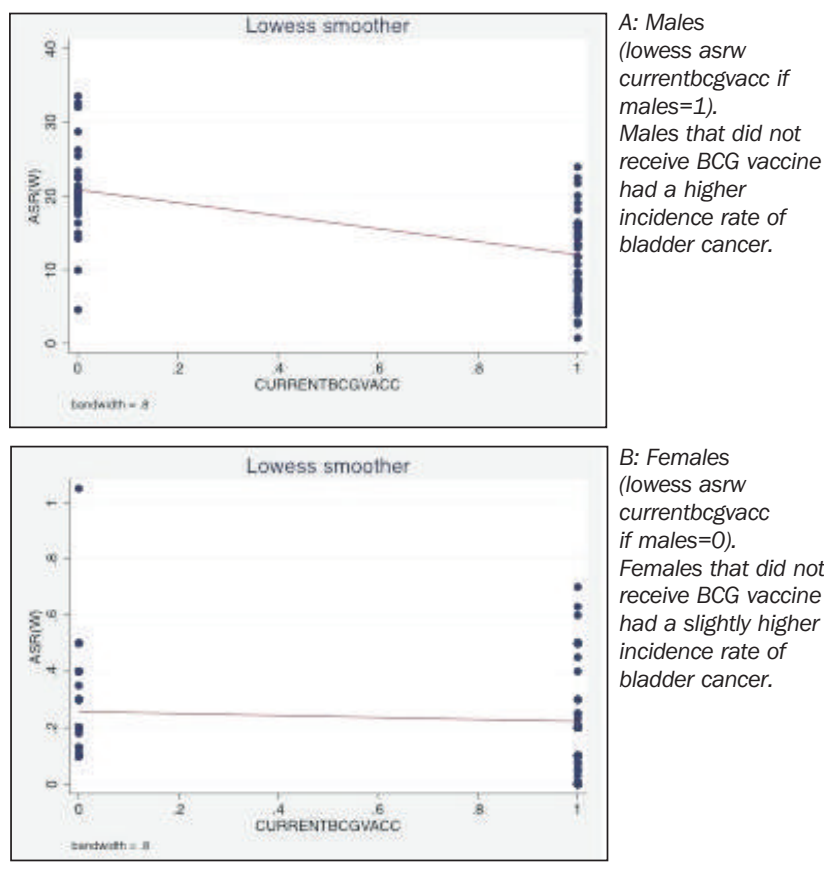

BCG vaccination [county has current BCG vaccination for tuberculosis ( 1 yes, 0 no)] while the second set uses never or past BCG vaccination [Country Never Had or Discontinued Universal BCG (1 yes, 0 no)] as the BCG variables. The third set uses whether the percent of 1 year olds vaccinated with BCG in 1985 was greater than 50\% [national share of $1 \mathrm{y}$ olds immunized (BCG) (WHO 2017 ) in $1985>50 \%$ ( 1 if $>50 \%, 0$ if $<50 \%$ or country

\section{Figure 2.}

Age standardized bladder cancer incidence rate (2003-07) versus median per capita GDP (2003-07).
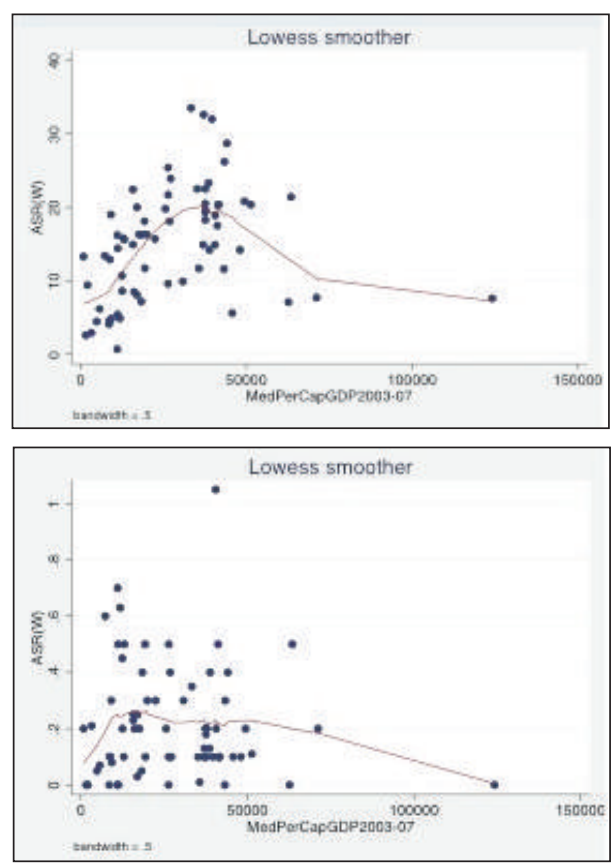

A: Males (lowess asrw medpercapgdp2003 07 if males $=1$, bwidth 0.5). There was a rise in the incidence rate of bladder cancer with increasing income to a certail level, after which, the incidence rate was declining.

B: Females (lowess asrw medpercapgdp2003 07 if females $=1$, bwidth 0.5).

There was a rise in the incidence rate of bladder cancer with increasing income to a certail level, after which, the incidence rate was declining 
never had or discontinued universal BCG = 1)]. The loglinear models are for the age-standardized rates only. They are of interest, because being log-linear, the coefficients can be interpreted as percentages and therefore allow for interpretation of magnitudes of the effects.

In general, the models suggest that gender, BCG vaccinations and socio-economic effects have statistically significant effects on crude and age-standardized rates of bladder cancer incidence at the 5 and 10 percent level.

The log-linear models suggest that all other things given, males have just over 4 times the rate of bladder cancer relative to females while the BCG vaccination variables are associated with a 35-37 percent lower age-standardized rate of bladder cancer incidence after controlling for both gender and GDP. When never having BCG or past $B C G$ TB vaccinations is the included vaccination variable, the results show a 20 percent higher rate of bladder cancer incidence, but the effect is not statistically significant. Meanwhile, controlling for gender and BCG vaccination, incidence rises and peaks at approximately $\$ 10,000$ in per capita GDP (in PPP\$) and then begins to decline.

There does seem to be some relationship between rates of TB vaccine inoculation and longer-term bladder cancer incidence in the results from this data.

These results are interesting but should be interpreted with extreme caution given the relatively small size of the data set as well as the single point in time nature of the data. These are only the 2003-07 incidence rates and the data set could be expanded to include the 2008-12 data. However, the ultimate limitation is the lack of fine granularity that would be provided by a much larger data set consisting of individuals over time - a time series microdata panel - with corresponding data on BCG vaccination and other individual level socio-economic characteristics.

Whether such a data set exists or could be constructed would be interesting.

Table 1 illustrate data retrieved from studies countries.

Table 1.

Details of countries included in our analysis. A table illustrating all retrieved data is included as a supplementary material.

\begin{tabular}{|c|c|c|c|c|c|c|c|c|c|c|c|c|c|c|}
\hline COUNTRY & $\begin{array}{l}\text { Total } \\
\text { cases }\end{array}$ & $\begin{array}{c}\text { Crude } \\
\text { rate }\end{array}$ & $\begin{array}{r}\text { ASR } \\
(W)\end{array}$ & M & $\mathbf{F}$ & $\begin{array}{l}\text { Current } \\
\text { BCG } \\
\text { vacc }\end{array}$ & $\begin{array}{l}\text { Never } \\
\text { or past } \\
\text { BCG }\end{array}$ & $\begin{array}{c}\text { Percent BCG } \\
1 \text { yr old } \\
2015>80 \%\end{array}$ & $\begin{array}{c}\text { Percent BCG } \\
1 \text { yr old } \\
1985\end{array}$ & $\begin{array}{c}\text { Percent BCG } \\
1 \text { yr old } \\
1985>50 \%\end{array}$ & $\begin{array}{c}\text { Percent BCG } \\
1 \text { yr old } \\
2015\end{array}$ & $\begin{array}{l}\text { MedPer } \\
\text { Cap GDP } \\
2003-07\end{array}$ & $\begin{array}{c}\text { Med } \\
\text { unemployment } \\
\text { rate 2003-07 }\end{array}$ & $\begin{array}{c}\text { MedG/GDP } \\
\text { 2003-07 }\end{array}$ \\
\hline \multicolumn{15}{|l|}{ MALES } \\
\hline Algeria & 203 & 5.60 & 8.60 & 1 & 0 & 1 & 0 & 1 & 86 & 1 & 99 & 12495.6 & 15.265 & 30.765 \\
\hline Argentina & 1238 & 15.75 & 14.93 & 1 & 0 & 1 & 0 & 1 & 90 & 1 & 99 & 15741.187 & 11.575 & 24.426 \\
\hline Australia & 12300 & 21.38 & 14.90 & 1 & 0 & 0 & 1 & 0 & & 0 & & 40460.543 & 5.042 & 34.681 \\
\hline Austria & 7271 & 36.40 & 20.30 & 1 & 0 & 0 & 1 & 0 & 90 & 1 & & 41363.055 & 5.225 & 50.378 \\
\hline Bahrain & 77 & 6.60 & 11.60 & 1 & 0 & & & & & & & 43218.759 & 5.6 & 24.923 \\
\hline Belarus & 4406 & 19.30 & 14.40 & 1 & 0 & 1 & 0 & 1 & & 0 & 97 & 11173.37 & 1.704 & 46.515 \\
\hline Belgium & 12617 & 61.50 & 32.00 & 1 & 0 & 0 & 1 & 0 & & 0 & & 39592.405 & 8.267 & 48.933 \\
\hline Brazil & 3906 & 7.38 & 10.68 & 1 & 0 & 1 & 0 & 1 & 63 & 1 & 99 & 12424.455 & 11.5 & 39.239 \\
\hline Bulgaria & 5237 & 27.90 & 15.60 & 1 & 0 & 1 & 0 & 1 & 99 & 1 & 97 & 13086.906 & 10.177 & 34.301 \\
\hline Canada & 24778 & 31.00 & 18.90 & 1 & 0 & 0 & 1 & 0 & & 0 & & 40588.876 & 6.758 & 38.662 \\
\hline Chile & 267 & 7.17 & 8.07 & 1 & 0 & 1 & 0 & 1 & 96 & 1 & 93 & 16958.13 & 9.3 & 20.137 \\
\hline Columbia & 451 & 4.50 & 4.85 & 1 & 0 & 1 & 0 & 1 & 76 & 1 & 90 & 9286.189 & 12.042 & 28.103 \\
\hline Costa Rica & 486 & 4.50 & 5.40 & 1 & 0 & 1 & 0 & 1 & 82 & 1 & 83 & 11110.728 & 6.495 & 16.658 \\
\hline Croatia & 3338 & 31.20 & 18.10 & 1 & 0 & 1 & 0 & 1 & & 0 & 98 & 19252.067 & 17.583 & 46.815 \\
\hline$\overline{\text { Cuba }}$ & 235 & 14.30 & 8.30 & 1 & 0 & 1 & 0 & 1 & 98 & 1 & 99 & & & \\
\hline Cyprus & 645 & 34.30 & 22.50 & 1 & 0 & 0 & 0 & 0 & & 0 & & 35179.581 & 4.55 & 39.127 \\
\hline Czech Rep & 8094 & 32.40 & 19.80 & 1 & 0 & 0 & 0 & 0 & & 0 & 98 & 25577.306 & 7.77 & 42.273 \\
\hline Denmark & 6464 & 48.20 & 26.20 & 1 & 0 & 0 & 1 & 0 & 80 & 1 & & 43342.624 & 4.8 & 51.242 \\
\hline Ecuador & 190 & 3.65 & 4.55 & 1 & 0 & 0 & 1 & 1 & 99 & 1 & 88 & 8616.508 & 7.095 & 21.213 \\
\hline Egypt & 1231 & 12.30 & 19.00 & 1 & 0 & 1 & 0 & 1 & 80 & 1 & 96 & 9132.877 & 10.917 & 30.638 \\
\hline Estonia & 763 & 24.60 & 15.70 & 1 & 0 & 1 & 0 & 1 & 90 & 1 & 95 & 22409.357 & 8.031 & 33.771 \\
\hline Finland & 3358 & 26.10 & 14.20 & 1 & 0 & 0 & 1 & 0 & 83 & 1 & & 38798.265 & 8.475 & 48.331 \\
\hline France & 7627 & 36.80 & 19.36 & 1 & 0 & 0 & 0 & 0 & 82 & 1 & & 37551.869 & 8.825 & 52.985 \\
\hline Germany & 24476 & 47.88 & 23.31 & 1 & 0 & 0 & 0 & 0 & & 0 & & 38597.366 & 10.042 & 46.791 \\
\hline Iran & 133 & 5.40 & 8.50 & 1 & 0 & 1 & 0 & 1 & 79 & 1 & 99 & 16089.323 & 11.3 & 19.848 \\
\hline Ireland & 2009 & 19.40 & 14.20 & 1 & 0 & 1 & 0 & 0 & 80 & 1 & 77 & 48034.889 & 4.775 & 33.343 \\
\hline Israel & 5033 & 30.60 & 25.40 & 1 & 0 & 0 & 1 & 0 & 68 & 1 & & 26340.356 & 11.2 & 43.685 \\
\hline Italy & 35154 & 68.32 & 32.61 & 1 & 0 & 0 & 1 & 0 & & 0 & & 37066.895 & 7.692 & 47.144 \\
\hline Jamaica & 58 & 3.70 & 4.10 & 1 & 0 & 1 & 0 & 1 & 51 & 1 & 99 & 8549.17 & 11.225 & 31.349 \\
\hline Japan & 10877 & 26.78 & 11.69 & 1 & 0 & 1 & 0 & 1 & 85 & 1 & 84 & 35664.003 & 4.425 & 34.598 \\
\hline Kuwait & 87 & 3.60 & 7.70 & 1 & 0 & 1 & 0 & 1 & & 0 & 99 & 71142.748 & 1.37 & 31.914 \\
\hline Latvia & 1040 & 24.70 & 16.30 & 1 & 0 & 1 & 0 & 1 & & 0 & 94 & 17601.947 & 10.05 & 33.478 \\
\hline
\end{tabular}




\begin{tabular}{|c|c|c|c|c|c|c|c|c|c|c|c|c|c|c|}
\hline Libya & 174 & 7.30 & 14.90 & 1 & 0 & 1 & 0 & 1 & 87 & 1 & 99 & 36946.588 & & 33.699 \\
\hline Lithuania & 1930 & 24.20 & 16.30 & 1 & 0 & 1 & 0 & 1 & & 0 & 97 & 18443.757 & 8.324 & 33.424 \\
\hline Malawi & 139 & 5.90 & 13.30 & 1 & 0 & 1 & 0 & 1 & 87 & 1 & 90 & 802.359 & & 26.394 \\
\hline Malaysia & 313 & 5.45 & 7.18 & 1 & 0 & 1 & 0 & 1 & 95 & 1 & 99 & 18241.829 & 3.55 & 25.884 \\
\hline Malta & 390 & 38.90 & 23.90 & 1 & 0 & 1 & 0 & 0 & 65 & 1 & & 27147.829 & 6.942 & 42.322 \\
\hline New Zealand & 1617 & 16.00 & 9.90 & 1 & 0 & 0 & 0 & 0 & & 0 & & 30803.692 & 3.875 & 37.066 \\
\hline Norway & 4467 & 38.90 & 21.40 & 1 & 0 & 0 & 0 & 0 & & 0 & & 63455.303 & 4.471 & 41.485 \\
\hline Phillipines & 622 & 2.05 & 4.45 & 1 & 0 & 1 & 0 & 0 & 76 & 1 & 80 & 4835.821 & 11.35 & 19.532 \\
\hline Poland & 4843 & 29.40 & 20.00 & 1 & 0 & 1 & 0 & 1 & 95 & 1 & 94 & 16982.21 & 17.745 & 44.395 \\
\hline Portugal & 174 & 29.00 & 21.70 & 1 & 0 & 1 & 0 & 0 & 82 & 1 & 32 & 26384.19 & 7.582 & 45.31 \\
\hline Qatar & 19 & 3.80 & 7.60 & 1 & 0 & 1 & 0 & 1 & 76 & 1 & 97 & 124151.906 & & 28.727 \\
\hline Korea & 11962 & 9.80 & 9.60 & 1 & 0 & 1 & 0 & 1 & 47 & 0 & 98 & 26340.46 & 3.55 & 19.202 \\
\hline Russia & 1745 & 17.00 & 11.70 & 1 & 0 & 1 & 0 & 1 & & 0 & 96 & 19374.661 & 7.15 & 29.598 \\
\hline Saudi Arabia & 266 & 2.70 & 5.60 & 1 & 0 & 1 & 0 & 1 & 89 & 1 & 98 & 45699.25 & 5.822 & 29.453 \\
\hline$\overline{\text { Serbia }}$ & 3997 & 30.30 & 16.20 & 1 & 0 & 1 & 0 & 1 & & 0 & 98 & 11017.344 & 19.53 & 41.9 \\
\hline Singapore & 680 & 7.90 & 7.10 & 1 & 0 & 1 & 0 & 1 & 93 & 1 & 99 & 62638.987 & 3.125 & 12.379 \\
\hline Slovakia & 2810 & 21.50 & 16.30 & 1 & 0 & 0 & 0 & 0 & & 0 & 90 & 19940.36 & 16.358 & 38.762 \\
\hline Slovenia & 1457 & 29.60 & 18.10 & 1 & 0 & 0 & 0 & 0 & 93 & 1 & & 26804.884 & 6.342 & 41.814 \\
\hline South Africa & 12 & 0.50 & 0.70 & 1 & 0 & 1 & 0 & 0 & 70 & 1 & 69 & 10999.622 & 24.65 & 27.175 \\
\hline Spain & 16120 & 61.37 & 33.52 & 1 & 0 & 0 & 1 & 0 & & 0 & & 33299.088 & 9.153 & 38.315 \\
\hline Sweden & 8256 & 36.80 & 17.50 & 1 & 0 & 0 & 1 & 0 & 16 & 0 & 24 & 41206.175 & 7.042 & 51.302 \\
\hline$\overline{\text { Switzerland }}$ & 4108 & 38.77 & 20.38 & 1 & 0 & 0 & 1 & 0 & & 0 & & 51299.143 & 3.691 & 32.896 \\
\hline Thailand & 1388 & 5.07 & 4.90 & 1 & 0 & 1 & 0 & 1 & 80 & 1 & 99 & 11772.887 & 1.858 & 19.331 \\
\hline Netherlands & 1243 & 48.00 & 28.70 & 1 & 0 & 0 & 1 & 0 & & 0 & & 44098.7 & 5.014 & 42.135 \\
\hline Tunisia & 883 & 12.00 & 12.90 & 1 & 0 & 1 & 0 & 1 & 90 & 1 & 97 & 8802.959 & 12.819 & 24.166 \\
\hline Turkey & 3607 & 23.58 & 22.43 & 1 & 0 & 1 & 0 & 1 & 76 & 1 & 95 & 15744.75 & 9.488 & 34.31 \\
\hline Uganda & 31 & 0.70 & 2.60 & 1 & 0 & 1 & 0 & 1 & 37 & 0 & 93 & 1559.811 & & 18.776 \\
\hline UK (England) & 46707 & 37.70 & 19.60 & 1 & 0 & 0 & 1 & 0 & & 0 & 75 & 37585.365 & 5 & 37.943 \\
\hline UK, Northern Ireland & 1250 & 29.60 & 18.30 & 1 & 0 & 0 & 1 & 0 & & 0 & 75 & 37585.365 & 5 & 37.943 \\
\hline UK, Scotland & 5271 & 42.90 & 22.50 & 1 & 0 & 0 & 1 & 0 & & 0 & 75 & 37585.365 & 5 & 37.943 \\
\hline UK, Wales & 3103 & 43.10 & 20.50 & 1 & 0 & 0 & 1 & 0 & & 0 & 75 & 37585.365 & 5 & 37.943 \\
\hline Ukraine & 21497 & 19.80 & 13.40 & 1 & 0 & 1 & 0 & 0 & & 0 & 39 & 7300.773 & 7.185 & 43.658 \\
\hline Uruguay & 1103 & 23.00 & 15.80 & 1 & 0 & 1 & 0 & 1 & 92 & 1 & 98 & 12872.245 & 12.142 & 29.186 \\
\hline USA & 60100 & 29.50 & 20.80 & 1 & 0 & 0 & 1 & 0 & & 0 & & 49412.962 & 5.083 & 33.931 \\
\hline Zimbabwe & 75 & 2.60 & 9.40 & 1 & 0 & 1 & 0 & 1 & 76 & 1 & 90 & 2065.22 & & 8.542 \\
\hline \multicolumn{15}{|l|}{ FEMALES } \\
\hline Algeria & 6 & 0.20 & 0.20 & 0 & 1 & 1 & 0 & 1 & 86 & 1 & 99 & 12495.6 & 15.265 & 30.765 \\
\hline Argentina & 5 & 0.15 & 0.23 & 0 & 1 & 1 & 0 & 1 & 90 & 1 & 99 & 15741.187 & 11.575 & 24.426 \\
\hline Australia & 1204 & 1.87 & 1.05 & 0 & 1 & 0 & 1 & 0 & & 0 & & 40460.543 & 5.042 & 34.681 \\
\hline$\overline{\text { Austria }}$ & 49 & 0.20 & 0.10 & 0 & 1 & 0 & 1 & 0 & 90 & 1 & & 41363.055 & 5.225 & 50.378 \\
\hline Bahrain & 0 & 0.00 & 0.00 & 0 & 1 & & & & & & & 43218.759 & 5.6 & 24.923 \\
\hline Belarus & 310 & 1.20 & 0.50 & 0 & 1 & 1 & 0 & 1 & & 0 & 97 & 11173.37 & 1.704 & 46.515 \\
\hline Belgium & 73 & 0.30 & 0.10 & 0 & 1 & 0 & 1 & 0 & & 0 & & 39592.405 & 8.267 & 48.933 \\
\hline Brazil & 159 & 0.37 & 0.45 & 0 & 1 & 1 & 0 & 1 & 63 & 1 & 99 & 12424.455 & 11.5 & 39.239 \\
\hline Bulgaria & 260 & 1.30 & 0.50 & 0 & 1 & 1 & 0 & 1 & 99 & 1 & 97 & 13086.906 & 10.177 & 34.301 \\
\hline Canada & 395 & 0.50 & 0.20 & 0 & 1 & 0 & 1 & 0 & & 0 & & 40588.876 & 6.758 & 38.662 \\
\hline$\overline{C h i l e}$ & 1 & 0.03 & 0.03 & 0 & 1 & 1 & 0 & 1 & 96 & 1 & 93 & 16958.13 & 9.3 & 20.137 \\
\hline$\overline{C h i n a}$ & 78 & 0.09 & 0.07 & 0 & 1 & 1 & 0 & 1 & 64 & 1 & 99 & 5669.225 & 4.2 & 18.074 \\
\hline Columbia & 14 & 0.10 & 0.08 & 0 & 1 & 1 & 0 & 1 & 76 & 1 & 90 & 9286.189 & 12.042 & 28.103 \\
\hline Costa Rica & 1 & 0.00 & 0.00 & 0 & 1 & 1 & 0 & 1 & 82 & 1 & 83 & 11110.728 & 6.495 & 16.658 \\
\hline Croatia & 133 & 1.20 & 0.50 & 0 & 1 & 1 & 0 & 1 & & 0 & 98 & 19252.067 & 17.583 & 46.815 \\
\hline Cuba & 11 & 0.70 & 0.50 & 0 & 1 & 1 & 0 & 1 & 98 & 1 & 99 & & & \\
\hline$\overline{C y p r u s}$ & 4 & 0.20 & 0.10 & 0 & 1 & 0 & 0 & 0 & & 0 & & 35179.581 & 4.55 & 39.127 \\
\hline Czech Rep & 153 & 0.60 & 0.20 & 0 & 1 & 0 & 0 & 0 & & 0 & 98 & 25577.306 & 7.77 & 42.273 \\
\hline Denmark & 87 & 0.60 & 0.30 & 0 & 1 & 0 & 1 & 0 & 80 & 1 & & 43342.624 & 4.8 & 51.242 \\
\hline Ecuador & 5 & 0.10 & 0.10 & 0 & 1 & 0 & 1 & 1 & 99 & 1 & 88 & 8616.508 & 7.095 & 21.213 \\
\hline Egypt & 16 & 0.20 & 0.30 & 0 & 1 & 1 & 0 & 1 & 80 & 1 & 96 & 9132.877 & 10.917 & 30.638 \\
\hline Estonia & 32 & 0.90 & 0.30 & 0 & 1 & 1 & 0 & 1 & 90 & 1 & 95 & 22409.357 & 8.031 & 33.771 \\
\hline Finland & 161 & 1.20 & 0.40 & 0 & 1 & 0 & 1 & 0 & 83 & 1 & & 38798.265 & 8.475 & 48.331 \\
\hline France & 88 & 0.46 & 0.18 & 0 & 1 & 0 & 0 & 0 & 82 & 1 & & 37551.869 & 8.825 & 52.985 \\
\hline
\end{tabular}




\begin{tabular}{|c|c|c|c|c|c|c|c|c|c|c|c|c|c|c|}
\hline Germany & 181 & 0.40 & 0.13 & 0 & 1 & 0 & 0 & 0 & 0 & 0 & & 38597.366 & 10.042 & 46.791 \\
\hline Iceland & 2 & 0.30 & 0.10 & 0 & 1 & 0 & & 0 & & 0 & & 41633.012 & 2.875 & 41.303 \\
\hline India & 186 & 0.18 & 0.21 & 0 & 1 & 1 & 0 & 1 & 8 & 0 & 87 & 3258.168 & & 26.659 \\
\hline Iran & 5 & 0.20 & 0.20 & 0 & 1 & 1 & 0 & 1 & 79 & 1 & 99 & 16089.323 & 11.3 & 19.848 \\
\hline Ireland & 19 & 0.20 & 0.10 & 0 & 1 & 1 & 0 & 0 & 80 & 1 & 77 & 48034.889 & 4.775 & 33.343 \\
\hline |srael & 32 & 0.20 & 0.10 & 0 & 1 & 0 & 1 & 0 & 68 & 1 & & 26340.356 & 11.2 & 43.685 \\
\hline Italy & 216 & 0.43 & 0.13 & 0 & 1 & 0 & 1 & 0 & & 0 & & 37066.895 & 7.692 & 47.144 \\
\hline Jamaica & 1 & 0.10 & 0.00 & 0 & 1 & 1 & 0 & 1 & 51 & 1 & 99 & 8549.17 & 11.225 & 31.349 \\
\hline Japan & 47 & 0.11 & 0.01 & 0 & 1 & 1 & 0 & 1 & 85 & 1 & 84 & 35664.003 & 4.425 & 34.598 \\
\hline Kuwait & 2 & 0.10 & 0.20 & 0 & 1 & 1 & 0 & 1 & & 0 & 99 & 71142.748 & 1.37 & 31.914 \\
\hline Latvia & 26 & 0.50 & 0.20 & 0 & 1 & 1 & 0 & 1 & & 0 & 94 & 17601.947 & 10.05 & 33.478 \\
\hline Libya & 1 & 0.00 & 0.10 & 0 & 1 & 1 & 0 & 1 & 87 & 1 & 99 & 36946.588 & & 33.699 \\
\hline Lithuania & 91 & 1.00 & 0.40 & 0 & 1 & 1 & 0 & 1 & & 0 & 97 & 18443.757 & 8.324 & 33.424 \\
\hline $\begin{array}{l}\text { Malawi } \\
\end{array}$ & 3 & 0.10 & 0.20 & 0 & 1 & 1 & 0 & 1 & 87 & 1 & 90 & 802.359 & & 26.394 \\
\hline$\overline{\text { Malaysia }}$ & 4 & 0.08 & 0.05 & 0 & 1 & 1 & 0 & 1 & 95 & 1 & 99 & 18241.829 & 3.55 & 25.884 \\
\hline Malta & 2 & 0.20 & 0.10 & 0 & 1 & 1 & 0 & 0 & 65 & 1 & & 27147.829 & 6.942 & 42.322 \\
\hline $\begin{array}{l}\text { New Zealand } \\
\end{array}$ & 47 & 0.40 & 0.30 & 0 & 1 & 0 & 0 & 0 & & 0 & & 30803.692 & 3.875 & 37.066 \\
\hline Noway & 139 & 1.20 & 0.50 & 0 & 1 & 0 & 0 & 0 & & 0 & & 63455.303 & 4.471 & 41.485 \\
\hline Phillipines & 8 & 0.00 & 0.05 & 0 & 1 & 1 & 0 & 0 & 76 & 1 & 80 & 4835.821 & 11.35 & 19.532 \\
\hline$\overline{\text { Poland }}$ & 106 & 0.55 & 0.25 & 0 & 1 & 1 & 0 & 1 & 95 & 1 & 94 & 16982.21 & 17.745 & 44.395 \\
\hline Portugal & 8 & 1.30 & 0.50 & 0 & 1 & 1 & 0 & 0 & 82 & 1 & 32 & 26384.19 & 7.582 & 45.31 \\
\hline$\overline{\text { Qatar }}$ & 0 & 0.00 & 0.00 & 0 & 1 & 1 & 0 & 1 & 76 & 1 & 97 & 124151.906 & & 28.727 \\
\hline Korea & 64 & 0.10 & 0.00 & 0 & 1 & 1 & 0 & 1 & 47 & 0 & 98 & 26340.46 & 3.55 & 19.202 \\
\hline Russia & 41 & 0.30 & 0.10 & 0 & 1 & 1 & 0 & 1 & & 0 & 96 & 19374.661 & 7.15 & 29.598 \\
\hline Saudi Arabia & 4 & 0.00 & 0.10 & 0 & 1 & 1 & 0 & 1 & 89 & 1 & 98 & 45699.25 & 5.822 & 29.453 \\
\hline Serbia & 195 & 1.40 & 0.70 & 0 & 1 & 1 & 0 & 1 & & 0 & 98 & 11017.344 & 19.53 & 41.9 \\
\hline Singapore & 4 & 0.00 & 0.00 & 0 & 1 & 1 & 0 & 1 & 93 & 1 & 99 & 62638.987 & 3.125 & 12.379 \\
\hline Slovakia & 100 & 0.70 & 0.30 & 0 & 1 & 0 & 0 & 0 & & 0 & 90 & 19940.36 & 16.358 & 38.762 \\
\hline Slovenia & 51 & 1.00 & 0.40 & 0 & 1 & 0 & 0 & 0 & 93 & 1 & & 26804.884 & 6.342 & 41.814 \\
\hline South Africa & 0 & 0.00 & 0.00 & 0 & 1 & 1 & 0 & 0 & 70 & 1 & 69 & 10999.622 & 24.65 & 27.175 \\
\hline Spain & 242 & 0.88 & 0.35 & 0 & 1 & 0 & 1 & 0 & & 0 & & 33299.088 & 9.153 & 38.315 \\
\hline Sweden & 286 & 1.30 & 0.50 & 0 & 1 & 0 & 1 & 0 & 16 & 0 & 24 & 41206.175 & 7.042 & 51.302 \\
\hline Switzerland & 28 & 0.21 & 0.11 & 0 & 1 & 0 & 1 & 0 & & 0 & & 51299.143 & 3.691 & 32.896 \\
\hline Thailand & 163 & 0.72 & 0.63 & 0 & 1 & 1 & 0 & 1 & 80 & 1 & 99 & 11772.887 & 1.858 & 19.331 \\
\hline Netherlands & 325 & 0.80 & 0.40 & 0 & 1 & 0 & 1 & 0 & & 0 & & 44098.7 & 5.014 & 42.135 \\
\hline Tunisia & 5 & 0.10 & 0.10 & 0 & 1 & 1 & 0 & 1 & 90 & 1 & 97 & 8802.959 & 12.819 & 24.166 \\
\hline Turkey & 49 & 0.33 & 0.25 & 0 & 1 & 1 & 0 & 1 & 76 & 1 & 95 & 15744.75 & 9.488 & 34.31 \\
\hline Uganda & 0 & 0.00 & 0.00 & 0 & 1 & 1 & 0 & 1 & 37 & 0 & 93 & 1559.811 & & 18.776 \\
\hline UK (England) & 371 & 0.30 & 0.10 & 0 & 1 & 0 & 1 & 0 & & 0 & 75 & 37585.365 & 5 & 37.943 \\
\hline UK, Northern Ireland & 17 & 0.40 & 0.20 & 0 & 1 & 0 & 1 & 0 & & 0 & 75 & 37585.365 & 5 & 37.943 \\
\hline UK, Scotland & 60 & 0.50 & 0.20 & 0 & 1 & 0 & 1 & 0 & & 0 & 75 & 37585.365 & 5 & 37.943 \\
\hline UK, Wales & 26 & 0.30 & 0.20 & 0 & 1 & 0 & 1 & 0 & & 0 & 75 & 37585.365 & 5 & 37.943 \\
\hline Ukraine & 1871 & 1.50 & 0.60 & 0 & 1 & 1 & 0 & 0 & & 0 & 39 & 7300.773 & 7.185 & 43.658 \\
\hline Uruguay & 10 & 0.20 & 0.10 & 0 & 1 & 1 & 0 & 1 & 92 & 1 & 98 & 12872.245 & 12.142 & 29.186 \\
\hline USA & 702 & 0.30 & 0.20 & 0 & 1 & 0 & 1 & 0 & & 0 & & 49412.962 & 5.083 & 33.931 \\
\hline Zimbabwe & 0 & 0.00 & 0.00 & 0 & 1 & 1 & 0 & 1 & 76 & 1 & 90 & 2065.22 & & 8.542 \\
\hline
\end{tabular}

\section{Discussion}

There was one study we identified in our scoping literature review that discussed the relationship between childhood BCG vaccination and subsequent development of lung cancer and secondarily, bladder cancer development. The study occurred at 9 sites in 5 American states between 1935 and 1998 and involved Indigenous schoolchildren with no prior evidence of TB infection. One cohort received a single intradermal BCG injection and the other a saline placebo

BCG vaccination was found not to be associated with a decreased rate of bladder cancer. The authors, however, noted significant limitations to their findings given their small sample size, and therefore this association may be masked (14). Findings of our literature search yielding 1 article was expected and indicate that additional studies are required in this area. In support of future studies examining the relationship between bladder cancer development and previous BCG vaccination, we performed a preliminary exploration of data, the results of which were discussed above.

\section{Racial variation of bladder cancer}

Bladder cancer is the $9^{\text {th }}$ most common malignancy, with five hundred and fifty thousand new cases annually worldwide. Geographically, there is significant variation 
in the incidence of NMIBC. Asian countries are found to have the lowest incidence of bladder cancer, while North American and Western European countries have the highest rates. Pursuant to our previous discussion, Asian countries have universal BCG vaccination programs, while many western countries do not. The incidence of bladder cancer also tends to increase as GDP increases, before levelling off and declining at higher levels of GDP. There are also racial and ethnic variations in the incidence of bladder cancer where it is twice as likely to occur in white males compared to African American or Hispanic men (15).

\section{BCG vaccination worldwide}

In 2010, a database was compiled of immunization protocols; among the 180 countries with available data, 157 countries recommend universal BCG vaccination, while the remaining 23 countries have either stopped BCG vaccination due to a reduction in TB incidence, or never recommended mass BCG immunization and instead favored selective vaccination of "at risk" groups.

The United States and Canada only recommended BCG immunization for high-risk groups and do not advocate for universal BCG vaccination. In contrast, other countries such as the United Kingdom had universal vaccination programs against TB.

The M. tuberculosis genome was initially published in 1921, and since this time comparative genomic studies have demonstrated the evolution of BCG vaccine strains. In other words, there are genetic differences in the antigenic proteins utilized in different vaccines over the years, which may translate into variations in efficacy over time. In spite of these differences, we have observed a $35-37 \%$ lower age-standardized rate of bladder cancer incidence in countries that vaccinate with the BCG vaccine, even after controlling for both gender and GDP (17).

\section{Early childhood BCG vaccination and its anti-neoplastic effects}

Although the BCG vaccine is efficacious against childhood $\mathrm{TB}$, its efficacy would be expected to diminish with time and provide variable protection in adulthood. Existing literature surrounding BCG immunization and leukemia gives some indication that long-lasting anti-neoplastic properties of the vaccine may exist, despite the waning immunity of the vaccine against TB. Specifically, these findings were summarized in a meta-analysis combining multiple studies. The aforementioned review indicated that early life-vaccination is associated with lower rates of childhood leukemia (17).

Another study demonstrated a reduced incidence of lung adenocarcinoma and squamous cell cancer with infantile BCG vaccination (14).

\section{Limitations}

The findings presented in this scoping literature review and preliminary data exploration should be interpreted with extreme caution given the relatively small data set as well as data giving a sole snapshot of statistics.

However, the ultimate limitation is the lack of fine granularity that would be provided by a much larger data set consisting of individuals over time, such as with a time series microdata panel. A time series microdata panel, which would ideally have corresponding data on BCG vaccination and other individual level socio-economic characteristics and co-founding variables for bladder carcinogenesis such as smoking.

\section{Conclusions}

Bladder cancer remains one of the most common malignancies globally. Prior work suggests there may be a protective relationship between BCG vaccination and rates of certain cancers.

This scoping literature review and preliminary data analysis provides a strong call for future work examining whether prior BCG vaccination correlates with a lower incidence of bladder cancer and whether this vaccine actually has a protective effect on the development of bladder malignancy.

\section{REFERENCES}

1. Raviglione MC, Snider DE, Kochi A. Global epidemiology of tuberculosis: Morbidity and mortality of a worldwide epidemic. JAMA. 1995; 273:220-226.

2. World Health Organization. Immunization, vaccines and biologicals: National programmes and systems. Retrieved (2020) from https://www.who.int/immunization/programmes_systems/en/\#: :text= The $\% 20$ EPI\%20launched\%20at\%20that,vaccine)\%2C\%20measles\%2 Oand\%20poliomyelitis.

3. Keja K, Chan C, Hayden G, Henderson RH. Expanded programme on immunization. World Health Stat Q. 1988; 41:59-63.

4. World Health Organization. BCG vaccine: WHO position paper, February 2018-recommendations. Vaccine. 2018; 24:3408-3410.

5. Faust L, Schreiber Y, Bocking N. A systematic review of BCG vaccination policies among high-risk groups in low TB-burden countries: implications for vaccination strategy in Canadian indigenous communities. BMC Public Health. 2019; 19:1504.

6. World Health Organization. Expanded programme on immunization (EPI) factsheet 2019: South-East Asia region.

7. Gillini L, Cooreman E, Wood T, et al. Global practices in regard to implementation of preventive measures for leprosy. PLoS Negl Trop Dis. 2017; 11:e0005399.

8. Taylor J, Becher E, Steinberg G. Update on the guideline of guidelines: Non muscle-invasive bladder cancer. BJUI. 2020; 125:197-205.

9. Moss JT, Kadmon, D. BCG and the treatment of superficial bladder cancer. DICP. 1991; 25:1355-1367.

10. Chen S, Zhang N, Shao J, Wang X. Maintenance versus non-maintenance intravesical bacillus calmette-guerin installation for non-muscle invasive bladder cancer: A systematic review and meta-analysis of randomized clinical trials. Intl JOS. 2018; 52:248-257.

11. Van Puffelen JH, Keating ST, Oosterwijk E, et al. Trained immunity as a molecular mechanism for BCG immunotherapy in bladder cancer. Nature Rev Uro. 2020; 17:513-525.

12. World Cancer Research Fund. Worldwide cancer data: Global cancer statistics for the most common cancers. Retrieved 82018) from https://www.wcrf.org/dietandcancer/cancer-trends/worldwide-cancerdata. 
13. Richters A, Aben KH, Kiemeney LA. The global burden of urinary bladder cancer: An update. World J Urol. 2020; 38:1895-1904

14. Usher NT, Chang S, Howard RS, et al. Association of BCG vaccination in childhood with subsequent cancer diagnoses: a 60-year follow-up of a clinical trial. JAMA Netw Open. 2019; 2:e1912014.

15. Daneshmand, Siamak. Epidemiology and risk factors of urothelial (transitional cell) carcinoma of the bladder. Retrieved (2020) from: https://www-uptodate-com.proxy.lib.nosm.ca/contents/epidemiologyand-risk-factors-of-urothelial-transitional-cell-carcinoma-of-the-blad- der?search=bladder\%20 cancer\%20epidemiologyEsource=search_resu ltEselectedTitle $=1 \sim 150 \varepsilon$ usage_type=default $\varepsilon$ display_rank=1\#H4.

16. Zwerling A, Behr M, Brewer T, et al. The BCG World Atlas: A Database of Global BCG Vaccination Policies and Practises. Retrieved (2011) from: https://www.ncbi.nlm.nih.gov/pmc/articles/PMC306 2527/

17. Morra ME, Kien ND, Elmaraezy A, et al. Early vaccination protects against childhood leukemia: A systematic review and meta-analysis. Sci Rep. 2017; 7:15986.

\section{Correspondence}

Sabrina Trigo,MD - strigo@nosm.ca

Kaitlin Gonzalez, MD - kquinlan@nosm.ca

Asmaa Ismail, MD - asmaaismail0782@gmail.com

Hazem Elmansy - hazemuro100@yahoo.com

Walid Shahrour - walid.shahrour@gmail.com

Owen Prowse - owenprowse@rogers.ca

Ahmed Kotb, MD (Corresponding Author)

drahmedfali@gmail.com

Assistant Professor Urology Department, Northern Ontario School of

Medicine, Thunder Bay Regional Health Science Centre

980 Oliver Rd, Thunder Bay, ON, Canada, P7B 6V4

Livio Di Matteo,PhD - 1dimatte@lakeheadu.ca

Department of Economics, Lakehead University, Thunder Bay, ON, Canada 